\title{
Focus Point on New Technologies Related to Intentional and Accidental Release of CBRNe Agents
}

\author{
Published online: 19 November 2018 - (c) Società Italiana di Fisica / Springer-Verlag GmbH Germany, part of
} Springer Nature, 2018

Since the events of September 11th 2001 and the anthrax attacks in America of the following month, the general consensus is that there is a realistic possibility of some form of unconventional terrorist attack in the Western World and that this could involve CBRN material (i.e., Chemical, Biological, Radioactive, Nuclear and explosive events). It is therefore vital that local authorities and agencies operating in the National and International frameworks are prepared to respond, as effectively and efficiently as possible, to any crisis scenarios resulting from such non-conventional events [1].

The loss of national control and the global spread of knowledge related to chemical, biological, radiological and nuclear weapons and technologies have been a long-standing concern for the post-Cold War World. In recent years, the fear that terrorist groups might employ CBRNe agents has particularly increased as some of these sensitive technologies and underpinning scientific knowledge have become more easily available for use in crude weapons. The National Security Strategy places strong emphasis on these concerns by including the risk of international terrorism activity with the possibility of using CBRNe agents at the highest priority level; the risk of CBRNe attacks from state actors ranks just one priority level below. Multidisciplinary research, focused on the long-term perspective, will play an important role in understanding the implications of constant rapid technological development in the CBRNe area. Furthermore, it might also enlighten on how the global spread of scientific education might affect aspirations of different State and non- State actors to use these technologies and knowledge for malevolent actions. A clearer understanding of these developments and the direction they may take will aid the progress of more effective policies and tools to counter possible CBRNe threats. Because of security budget reduction, the way in which different Countries prepare for CBRNe incidents deserves renewed attention; this involves the prioritization of capabilities against C, B, $\mathrm{R}$, or $\mathrm{N}$ in the Analysis, Prevention and Response (APR) phase [2]. It will also be necessary to acquire detailed information about the capability of the actor involved to use or produce CBRNe weapons, taking into account all the latest scientific developments in the field of chemistry, physics, biology and nanotechnology. An analysis of how actual CBRNe threats and hazards are perceived by policy makers from different Countries shows the following outcomes:

- There is consensus on the importance of CBRNe threats. Some Countries list CBRNe terrorism, or CBRNe weapon use and proliferation among the most important security threats.

- The general perception is that State actors have the potential to acquire CBRNe expertise and experts, but are restrained to deploy them; the opposite holds true for non-State actors.

With respect to science and technology, experts expect: a) an increasing interaction between chemistry and biology know-how development; b) dramatic advances in understanding and manipulating genes, cells, and organisms; and c) developments in the field of nanotechnology that may revolutionize dispersal methods.

The CBRNe world offers several starting points for national and international collaborations in a wide range of public, private, research, and industrial contexts. It is important to create the conditions to connect the best experts, allowing a reverse brain drain process. Why pursuing this challenge? To create a new way of working together and, above all, to have a new vision of work. It is essential to identify the needs in the CBRNe safety and security framework and then deploy existing skills and develop new theoretical and practical knowledge to answer those needs. 
This Focus Point collects some innovative tech solutions presented during the first international conference on CBRNe - SICC 2017, a conference devoted to promoting the dissemination of the different methodologies, techniques, theories, strategies, technologies and best practices on the prevention and mitigation of CBRNE risks. The conference intended to propose new solutions to reduce the risk factors related to CBRNe events and to promote the fruitful inter-professional collaborations between university and military/public experts, specialized operators, decision makers and the industry.

The concepts introduced in this Focus Point are directly elicited form the SICC 2017 conference and the papers should be read in this context. In this Focus Point, we explore the role that science and technology play in contributing to preventing and mitigating disasters involving CBRNe events, no matter whether they are caused by accidents or by terrorist acts.

In The impact of climate change on radiological practices in Italy - Safety implications and preventive measures [3], the authors analyze the impact of extreme weather events and climate-related disasters on a number of radiological activities. They conclude that specific adaptation strategies are needed to address the impact of climate change and that they should be included in the safety and emergency plans, according to the principle of optimization of radiation protection.

The paper entitled $\delta^{13} C$ analysis to screen out explosive precursors by using cavity ring down laser spectroscopy by Rizzo et al. [4] presents a new analytical platform to analyze isotope ratio of carbon $\delta^{13} \mathrm{C}$ and nitrogen content using a cavity ring down spectroscopy laser spectrometer.

The article by Pascual et al., titled First measurement using COUNTERFOG device: Nuclear and radiological scenario [5], focuses on the novel COUNTERFOG device, a countermeasure system based on the generation of a fog that interacts with dispersed aerosols. In their work they present the results of the application of the COUNTERFOG system to the cleaning of RN surrogates, dispersed in the environment simulating the detonation of a RDD device.

In Novel standoff detection system for the classification of chemical and biological hazardous substances combining temporal and spectral laser-induced fluorescence techniques [6], the authors present a novel detection system for the classification of chemical and biological hazardous substances.

In Laser-based optical techniques for the detection of chemical agents [7], Trtica and colleagues provide an interesting review of several laser-based systems for optical sensing of gasphase chemical agents, outlining principles of operation and analytical capabilities of such systems.

The article by Tsuchiya Radiation-detection methods to counter radiological threats [8] focuses on two methods to counter radiological attacks. The first method estimates the neutron radiation dose from the amount of noise in CCD images produced by surveillance cameras placed in public areas. The second method describes a survey system of radiological residue using long scintillation fibers.

Finally, D'Errico et al., in their paper Detection of special nuclear material with a transportable active interrogation system [9] report on a novel active interrogation system based on detectors containing liquid droplets that vaporize when exposed to fast neutrons and on an ultra-compact linear accelerator (LINAC). The system was successfully tested in the detection of special nuclear material.

Our special thanks go to all the editorial staff for their valuable and prompt support throughout the preparation and publication of this Focus Point. We would like to thank all authors for their contributions to this special issue. We also extend our thanks to all reviewers for their hard work to ensure the high quality of accepted papers.

\section{References}

1. F. Bruno et al., NATO Sci. Peace Secur. Ser. A: Chem. Biol. F1, 309 (2018).

2. A. Malizia et al., Def. Secur. Anal. 32, 79 (2016).

3. G.M. Contessa et al., Eur. Phys. J. Plus 133, 380 (2018).

4. A. Rizzo et al., Eur. Phys. J. Plus 133, 292 (2018).

5. Laura Pascual et al., Eur. Phys. J. Plus 133, 291 (2018).

6. Florian Gebert et al., Eur. Phys. J. Plus 133, 269 (2018).

7. Milan S. Trtica et al., Eur. Phys. J. Plus 133, 268 (2018).

8. Ken'ichi Tsuchiya et al., Eur. Phys. J. Plus 133, 266 (2018).

9. Francesco d'Errico et al., Eur. Phys. J. Plus 133, 451 (2018).

A. Malizia and M. D'Arienzo 